García, R., Ferrández, R, Sales, Ma . A. y Moliner, Mª O. (2006). Elaboración de instrumentos de medida de las actitudes y opiniones del profesorado universitario hacia la ética profesional docente y su papel como transmisor de valores. RELIEVE, v. 12, n. 1, p. 129-149. www.uv.es/RELIEVE/v12n1/RELIEVEv12n1_8.htm

\title{
REIIदVE
}

Revista ELectrónica de Investigación

y EValuación Educativa

\section{ELABORACIÓN DE INSTRUMENTOS DE MEDIDA DE LAS ACTITUDES Y OPINIONES DEL PROFESORADO UNIVERSITARIO HACIA LA ÉTICA PROFESIONAL DO- CENTE Y SU PAPEL COMO TRANSMISOR DE VALORES}

\section{[Elaboration of measurement instruments for attitudes and opinions} from university academic personnel towards teaching professional ethics and its role as values transmitter]
$\underline{\text { Article record }}$
About authors
$\underline{\text { HTML format }}$

\author{
por
}

Ficha del artículo

$\underline{\text { Sobre los autores }}$

Formato HTML

\begin{abstract}
From the research study - a compared analysis of the university academic personnel's attitudes and values towards professional ethics -, our objective was to discover whether the personnel members themselves consider that the professional ethical dimension in general, and the professional teaching ethics in particular, are essential to be a good teaching professional. In this article, we present the elaboration and validation process of the questionnaire that has been used, with which we hope to make some contribution towards clarifying aspects related with both professional teaching ethics and the ethics of professions within the university environment.
\end{abstract}

\section{Keywords}

Instruments validation, attitudes, values, professional ethics, professional teaching ethics, University, Exploratory Factorial Analysis.

\section{Resumen}

A partir de la investigación que hemos realizado sobre "Análisis comparado de las actitudes y valores del profesorado universitario ante la ética profesional", con el objetivo de averiguar si el propio profesorado considera que la dimensión ética profesional en general, y la ética profesional docente, en particular, son esenciales para ser un buen profesional de la docencia, presentamos, en este artículo, el proceso de elaboración y validación del cuestionario que se ha utilizado, con el que esperamos hacer alguna aportación que nos permita aclarar aspectos relacionados con la ética profesional docente y con la ética de las profesiones en el ámbito universitario.

\section{Descriptores}

Validación de instrumentos, actitudes, valores, ética profesional, ética profesional docente, Universidad, Análisis Factorial exploratorio. 
La convergencia europea de la Enseñanza Superior supone asumir nuevos retos referentes, algunos, al papel que ha de desempeñar el profesorado universitario, cuya función docente comienza a tener un mayor peso y un mayor reconocimiento. Recordemos que el profesorado es valorado, sobre todo, por su capacidad investigadora (Gross y Romanyá, 2003; García López et al., 2005), dejando muy atrás su capacidad docente, normalmente valorada únicamente por medio de encuestas realizadas por el alumnado, cuyos resultados tienen poca relevancia, tanto dentro como fuera de la institución. Centrar el esfuerzo en el aprendizaje del alumnado supone replantear la función docente realizada hasta el momento e incorporar innovaciones, tanto metodológicas como técnicas y éticas (Jover, Fernández y Ruiz, 2005).

Con el cuestionario, cuya validación presentamos en este artículo, esperamos hacer alguna aportación que nos permita aclarar aspectos relacionados con la ética profesional docente y con la ética de las profesiones en el ámbito universitario. Este estudio está dentro de la investigación que hemos realizado sobre "Análisis comparado de las actitudes y valores del profesorado universitario ante la ética profesional" realizada en el 2004-2005, ${ }^{[1]}$ con el objetivo de averiguar si el propio profesorado considera que la dimensión ética profesional en general, y la ética profesional docente, en particular, es esencial para ser un buen profesional de la docencia.

Pensamos que esta faceta de la formación del profesorado universitario está absolutamente descuidada en nuestra universidad (Zabalza, 2003; Bolívar, 2005); que existe la percepción de que los principios éticos no se aprenden a través de procesos formativos sistematizados, sino que es algo que se adquiere por la propia experiencia; que el profesorado parece que domina este campo lo suficiente como para no necesitar formación, etc. Todo esto requiere que repensemos hasta qué punto estamos preparados para asumir la enorme responsabilidad de ejercer, con los recursos necesarios (cognitivos, técnicos, metodológicos y éticos) la profesión docente. En resumen, pretendemos responder a las siguientes preguntas: ¿Qué cree el profesorado universitario que es la ética profesional docente?, ¿qué grado de importancia le concede?, ¿qué opina de los códigos deontológicos?, ¿debería existir uno para regular la docencia universitaria?, ¿qué ámbitos debería regular? y, por último ¿a qué le conceden más importancia?

\section{1. ÉTICA PROFESIONAL DOCEN- TE Y TRANSMISIÓN DE VALORES ÉTICOS}

Para entender la estructura y contenidos de los cuestionarios, nos parece oportuno realizar una pequeña clarificación conceptual sobre lo que significa ética profesional y ética profesional docente, puesto que, aunque tienen muchos puntos en común, es preciso matizar que no se trata de términos equivalentes.

Así, siguiendo a Hortal (2002), encontramos que el término ética se aplica a la conducta presumiblemente libre y responsable de una persona. Es una disciplina filosófica que investiga la conducta humana orientada hacia el bien. La ética general propone el cuadro de principios/valores básicos que han de servir de norte para que las personas puedan estructurar un proceder habitual en todos los órdenes. Por ejemplo, el principio y valor de la justicia, aplicable a todas las relaciones humanas. Por otra parte, están las éticas aplicadas, que introducen los principios de la ética general a ámbitos, problemas o a las actividades profesionales. En este caso hay que diferenciar entre Ética General de las Profesiones y Éticas profesionales concretas, es decir, la ética específica de cada profesión.

Entenderemos por ética profesional la disciplina que tiene por objeto determinar el conjunto de responsabilidades éticas y mora- 
García, R., Ferrández, R, Sales, Mª A. y Moliner, Mª O. (2006). Elaboración de instrumentos de medida de las actitudes y opiniones del profesorado universitario hacia la ética profesional docente y su papel como transmisor de valores. RELIEVE, v. 12, n. 1, p. 129-149. www.uv.es/RELIEVE/v12n1/RELIEVEv12n1_8.htm

les que surgen en relación al ejercicio de una profesión. Tanto la ética general de las profesiones como las éticas profesionales son éticas que en sus principios y directrices buscan el bien de los clientes o usuarios de los servicios, de la sociedad y de los propios profesionales (Cobo, 2001).

Si queremos aplicar la ética profesional a una determinada profesión, en este caso la docencia universitaria, es imprescindible aclarar sus funciones, puesto que junto a las funciones tradicionales han de ser completadas otras nuevas, entre las que hemos de valorar la educativa. Dentro de la docencia universitaria destacaríamos básicamente tres:

1. Formación integral del universitario: No podemos restringir exclusivamente la enseñanza universitaria al desarrollo de la inteligencia, pues olvidaríamos dimensiones básicas en la formación de la persona, como son: la dimensión afectiva y la dimensión moral y cívica.

2. Contribuir a crear una cultura universitaria para afrontar los problemas reales desde una visión relacionada, integradora $\mathrm{y}$ con vistas a un cambio que mejore y haga más justa nuestra sociedad (Esteban, 2004).

3. Contribuir a la implantación de la sociedad del conocimiento, pero desde la solidaridad.

A partir de estos conceptos fundamentales, y a través de diferentes dinámicas se han planteado y elaborado los cuestionarios que describimos en el presente trabajo.

\section{OBJETIVO}

Pretendemos estudiar las predisposiciones que el profesorado universitario tiene hacia las competencias éticas en el ejercicio de su profesión, como investigador y docente.

Nuestra investigación parte del convencimiento de la ausencia de preocupación de la institución universitaria acerca de la dimensión moral de su profesorado y de su alumnado. Tanto es así que en los procesos for- mativos del profesorado universitario prácticamente no se contemplan contenidos relacionados con la ética profesional y lo mismo ocurre con el alumnado. Se desarrollan competencias cognitivas, técnicas, etc., pero se descuida el desarrollo de las competencias éticas (Martínez, Buscarais y Esteban, 2002). Nuestra pregunta inicial es ¿hasta qué punto el profesorado universitario echa en falta su formación ética?

En la actualidad la Universidad afronta nuevos retos, no sólo por las derivadas de la Convergencia Europea, sino porque los cambios sufridos en nuestra sociedad demandan una universidad que incorpore la formación en valores éticos y deontológicos de sus miembros; porque se nos exige que formemos ciudadanos con una actitud responsable en su ejercicio profesional, comprometida con la libertad, la igualdad, la equidad, el respeto y la solidaridad. La universidad ha sido, desde sus orígenes, la encargada de formar profesionales en diversas áreas del conocimiento, y hoy debería ser la encargada de formar auténticos ciudadanos, responsables y comprometidos éticamente con la realidad social que les rodea (Escámez, Ortega y Martínez, 2005). Hemos de saber qué piensa de esto el profesorado, ¿cuál es su función como transmisor de valores éticos?

Este es el objetivo fundamental de nuestra investigación: realizar un estudio descriptivo de las actitudes, opiniones y expectativas del profesorado universitario hacia la ética profesional y hacia la formación en valores éticos de su alumnado para contribuir a desarrollar en ellos el sentido de ciudadanía. A diferencia de otros enfoques, proponemos conocer la realidad, mediante un estudio descriptivo, tal y como la perciben los implicados, para detectar mejor los problemas y dificultades de incorporar la dimensión ética en la función educativa del profesorado universitario y buscar soluciones que faciliten dicho objetivo para mejorar la calidad de la docencia universitaria. 


\section{DESCRIPCIÓN DE LOS INS- TRUMENTOS}

Para cumplir con este objetivo, se elaboró una batería de pruebas, compuesta por cuatro instrumentos:

I. Grupos de Discusión sobre ética profesional

II. Cuestionario sobre conocimientos, actitudes, opiniones y expectativas hacia la ética profesional docente en la enseñanza superior.

III. Cuestionario sobre valores que promueve la universidad y el profesorado.

IV. Cuestionario sobre valores del profesorado.

A continuación pasamos a describir cada uno de ellos

\subsection{Grupos de Discusión sobre ética profesional}

1. PLANTEAMIENTO DE OBJETIVOS Y ELABORACIÓN DE LA GUÍA DE PREGUNTAS

\section{Objetivos:}

a. Conocer las opiniones del profesorado hacia la ética profesional docente.

b. Detectar los valores que, a juicio del profesorado, promueve la Universidad en su alumnado y los que debería promover.

c. Conocer las percepciones del profesorado sobre su papel como educador en valores éticos y mediador del sentido de ciudadanía en su alumnado.

d. Detectar las opiniones de las profesoras y profesores sobre la necesidad o no de incluir en el currículo una materia sobre ética profesional.

\section{Guía de preguntas:}

1.a. ¿Qué competencias caracterizan a un buen docente universitario?

2.a. ¿Qué entienden por ética profesional docente? ¿A qué contenidos hace referencia? 3.a. ¿Creen que es necesario un código de ética profesional docente? ¿Qué ámbitos debería regular?

4.a. ¿Consideran importante la formación ética del profesorado para su buen ejercicio de la docencia y la investigación? ¿Por qué?

5.b. ¿Es una función de la Universidad enseñar valores a sus alumnos? ¿Qué valores consideran que debe transmitir la Universidad?

6.b. ¿Consideran que los valores de la ética profesional tienen algo que ver con los valores que debe transmitir la Universidad a sus alumnos?

7.c. ¿Es competencia del profesorado universitario transmitir valores éticos a sus alumnos?

8.c. ¿Debe ser el profesor universitario mediador del sentido de ciudadanía con sus alumnos?

9.d. ¿Consideran adecuado que existan asignaturas sobre ética profesional en todas las titulaciones?

10.d. ¿Qué contenidos consideran necesarios se deberían transmitir en una asignatura de este tipo?

\section{SELECCIÓN DE LOS PARTICIPAN- TES}

Los criterios para la selección fueron: a) pertenencia a diferentes áreas de conocimiento; b) en la medida de lo posible, con diferentes categorías profesionales y años de docencia, y ambos sexos. La muestra se seleccionó no de forma aleatoria, sino de forma intencional, "seleccionando a las personas según la relación que éstas guarden con el objeto de estudio" (Rubio y Varas, 1997:336).

En total participaron 24 profesores de diferentes categorías y áreas de conocimiento, con los que se formaron tres grupos de discusión atendiendo a la universidad de origen: Valencia, Madrid y Castellón. 
García, R., Ferrández, R, Sales, Mª A. y Moliner, Mª O. (2006). Elaboración de instrumentos de medida de las actitudes y opiniones del profesorado universitario hacia la ética profesional docente y su papel como transmisor de valores. RELIEVE, v. 12, n. 1, p. 129-149. www.uv.es/RELIEVE/v12n1/RELIEVEv12n1_8.htm

\section{SELECCIÓN DEL MODERADOR.}

Se seleccionó un moderador/a en cada universidad y una persona encargada del registro de la información para cada uno de los grupos de discusión.

\section{DETERMINACIÓN DEL LUGAR Y FECHA}

Las sesiones se efectuaron durante la primera semana de noviembre de 2004 y tuvieron una duración mínima de hora y media y máxima de dos horas. En ellas fueron grabadas, y posteriormente trascritas todas las intervenciones para su posterior análisis en fichas como las que se muestran en el punto siguiente.

\section{RECOPILACIÓN, TRASCRIPCIÓN Y ANÁLISIS DE LA INFORMACIÓN}

A continuación (Figura 1) se presenta un ejemplo de ficha de cada pregunta enunciada al comienzo del apartado 2.1 para que el o la secretaria fuera incorporando aspectos que considerara relevantes durante el desarrollo de la sesión. Posteriormente se realizó la trascripción, una clasificación de las respuestas en categorías relevantes, una descripción de cada una de ellas y la interpretación de los resultados.

Figura 1. Ejemplo de ficha de recogida de información relevante en los grupos de discusión

\begin{tabular}{|c|c|}
\hline TEMA & ÉTICA PROFESIONAL DOCENTE \\
\hline SUBTEMA & 1.a. ¿Qué competencias caracterizan a un buen docente universitario? \\
\hline \multicolumn{2}{|l|}{ OBSERVACIONES } \\
\hline \multicolumn{2}{|l|}{ FRASES } \\
\hline SUBTEMA & $\begin{array}{l}\text { 2.a. ¿Qué entienden por ética profesional docente? ¿A qué contenidos hace refe- } \\
\text { rencia? }\end{array}$ \\
\hline \multicolumn{2}{|l|}{ OBSERVACIONES } \\
\hline \multicolumn{2}{|l|}{ FRASES } \\
\hline SUBTEMA & $\begin{array}{l}\text { 3.a. ¿Creen que es necesario un código de ética profesional docente? ¿Qué ámbi- } \\
\text { tos debería regular? }\end{array}$ \\
\hline \multicolumn{2}{|l|}{ OBSERVACIONES } \\
\hline \multicolumn{2}{|l|}{ FRASES } \\
\hline SUBTEMA & $\begin{array}{l}\text { 4.a. ¿Consideran importante la formación ética del profesorado para su buen } \\
\text { ejercicio de la docencia y la investigación? ¿Por qué? }\end{array}$ \\
\hline \multicolumn{2}{|l|}{ OBSERVACIONES } \\
\hline FRASES & \\
\hline
\end{tabular}

\subsection{Cuestionario sobre conocimientos,} actitudes, opiniones y expectativas hacia la ética profesional docente en la enseñanza superior.

Este instrumento está dividido en dos partes: en la primera, que consta de ocho preguntas cerradas, se pretende obtener información sobre el grado de conocimiento y el grado de importancia que le conceden a la ética profesional docente. También se obtiene información sobre su opinión acerca de los códigos deontológicos, los ámbitos que han de regular y las principales competencias que caracterizan a un buen docente universitario. En la segunda parte, elaboramos una escala de actitudes, tipo Likert de cinco puntos, en la que pretendíamos medir sus actitudes hacia diferentes núcleos; los mismos que ya fueron previamente trabajados en los Grupos de Discusión ${ }^{[2]}$. En esta segunda parte, por tanto, se elaboró un CuestionarioEscala Piloto de Actitudes que incluía los siguientes núcleos: 
$1^{\circ}$ Actitudes hacia las competencias profesionales del docente universitario

Constaba de los 61 primeros ítems, distribuidos en los siguientes apartados:

a) Competencias cognitivas

- Conocimientos, formación y competencia profesional (Ítems 1 al 8)

- Preparación y formación continua (Ítems

9 al 16)

- Innovación y superación (Ítems 17 al 21)

b) Competencias técnico-organizativas

- Conocimiento y competencia técnica (Ítems 22 al 26)

c) Competencias sociales

- Compromiso y relaciones personales (Ítems 27 al 31)

- Comunicación (Ítems 32 al 38)

- Dedicación (Ítems 39 al 41)

- Saber trabajar en equipo (Ítems 42 al 46)

d) Competencias éticas

- Responsabilidad (Ítems 47 al 51)

- Honestidad (Ítems 52 al 53)

- Respeto (Ítems 54 al 55)

- Prestar servicio a la sociedad (Ítems 56 al 58)

e) Competencias afectivo-emocionales

- Identificarse con la profesión (Ítems 59

al 60)

- Capacidad emocional (61)

\section{$2^{\circ}$ Actitudes hacia la formación ética}

Consta de 12 ítems (del 62 al 74) que se elaboraron basándonos en las respuestas que el profesorado emitió a la pregunta 4.a de los Grupos de Discusión: ¿Considera importante la formación ética del profesorado para su buen ejercicio de la docencia y la investigación ¿ ¿Por qué?

\section{$3^{\circ}$ Actitudes hacia los códigos deontológi- $\cos$}

El resto de los ítems de este cuestionario, del 75 al 114, van referidos a las actitudes del profesorado universitario hacia la conveniencia o no de la existencia de códigos éticos para el ejercicio de la docencia en general (ítems 75 al 85) y más concretamente hacia los ámbitos específicos que regulan el ejercicio de la docencia, como son:

- Exigencias de uno mismo como profesional

- Deberes en las relaciones con los alumnos/as

- Relación con los compañeros

- Normativa relativa a la investigación

- Relación con la profesión o profesiones

- Relación con la sociedad

- Relación con la institución universitaria

Estos ítems también se elaboraron teniendo en cuenta las respuestas a la pregunta 3 a de los Grupos de Discusión: ¿Creen que es necesario un código de ética profesional docente? ¿Qué ámbitos debería regular?

\subsection{Cuestionario sobre valores que promueve la Universidad}

Este cuestionario también consta de dos partes: en la primera se realizan nueve preguntas, seis cerradas y tres abiertas sobre el papel que la universidad puede desempeñar en la enseñanza de valores a su alumnado; qué valores ha de desarrollar tanto en el alumnado como en el profesorado y qué dificultades se presentan para enseñar valores de una forma explícita. También aquí se incorporan dos preguntas sobre la conveniencia o no de incorporar una materia de ética profesional dirigida al alumnado en todas las titulaciones, así como los contenidos que, a juicio del profesorado, debería tener.

En la segunda parte se plantea una escala de actitudes del profesorado, compuesta de 30 ítems, hacia varios núcleos, trabajados previamente en los Grupos de Discusión:

a. Papel de la Universidad en la transmisión de valores (ítems 1 al 6). Procede de las respuestas a la pregunta $5 \mathrm{~b}$ de los Grupos de Discusión: ¿Es una función de la Universidad enseñar valores a sus 
García, R., Ferrández, R, Sales, Mª A. y Moliner, Ma . O. (2006). Elaboración de instrumentos de medida de las actitudes y opiniones del profesorado universitario hacia la ética profesional docente y su papel como transmisor de valores. RELIEVE, v. 12, n. 1, p. 129-149. www.uv.es/RELIEVE/v12n1/RELIEVEv12n1_8.htm

alumnos? ¿Qué valores considera que debe transmitir la Universidad?

b. Papel del profesorado en la enseñanza de valores y como mediador del sentido de ciudadanía. Los ítems se elaboraron atendiendo a las respuestas de los Grupos de Discusión de las preguntas 7c : ¿Es competencia del profesorado transmitir valores éticos a sus alumnos/as? Y 8c: $¿$ Debe ser el profesor universitario mediador del sentido de ciudadanía? En el cuestionario se plasman en los ítems 7 al 20, excepto el 14.

c. Formación del profesorado (ítems $14,21,22$ y 23 )

d. Asignatura de ética profesional. Responde a la pregunta 9d de los Grupos de Discusión: ¿Consideran adecuado que existan asignaturas sobre ética profesional en todas las titulaciones? En el cuestionario se plasman en los ítems 25 al 30 .

\subsection{Cuestionario sobre valores del pro- fesorado}

Pretendíamos conocer cómo se pronuncia el profesorado sobre una lista de valores relacionados con su ejercicio profesional. Para ello seleccionamos 24 valores, algunos seleccionados del instrumento de Rockeach (1973), aunque al final decidimos plantearlo en forma de escala tipo Thursthone, de siete grados, puesto que facilita la graduación de la respuesta y permite más matices en el análisis de los resultados. Los 24 valores se presentan con una breve definición, y le pedimos al profesorado que indiquen el grado de importancia (de 1 a 7) que le conceden a cada uno en relación con su ejercicio profesional docente. Los valores son: autoestima, competencia, responsabilidad, respeto, participación, sentido crítico, amistad, autonomía, bienestar, convivencia, cooperación, felicidad, constancia, libertad, sinceridad, diálogo, coherencia, civismo, flexibilidad, reconocimiento social, seguridad, innovación, liderazgo, perfectibilidad.

\section{PROCESO DE VALIDACIÓN}

El instrumento piloto constaba de 162 ítems divididos en tres grandes bloques independientes, además de un apartado inicial destinado a cuestiones de tipo personal con el fin de obtener mayor información sobre los respondientes. Para el estudio preliminar se recogieron respuestas de un total de 81 profesores de las universidades participantes en el estudio (Valencia Estudi General, Complutense de Madrid y Jaume I de Castellón), además de otras cercanas al contexto en el que se desarrolla esta investigación pero que no participan directamente.

El primero de los bloques estaba relacionado con las actitudes personales hacia las competencias que caracterizan a un buen docente y, especialmente hacia la ética profesional docente. Constaba de 114 preguntas a responder en una escala tipo Likert de cinco puntos.

El segundo bloque se destinaba a las actitudes hacia la universidad como ente transmisor de valores. Estaba formado por 30 ítems también estructurados para ser respondidas en una escala Likert de las mismas características que la anterior.

El tercer bloque constaba de 24 elementos cuyo contenido se destinaba a valorar la importancia de diferentes valores en el ejercicio profesional del docente universitario.

La depuración de los instrumentos se ha realizado considerando las tres partes como cuestionarios independientes, puesto que los objetivos de la investigación trataban estas tres partes de manera diferenciada y por tanto era preciso mantener la independencia para obtener información de todos los aspectos necesarios en el estudio posterior. El instrumento definitivo una vez depurado se muestra en el anexo al final de este artículo.

Las respuestas obtenidas fueron introducidas en el Paquete estadístico SPSS (versión 12.0) para proceder a la depuración y análisis 
García, R., Ferrández, R, Sales, Mª A. y Moliner, Mª O. (2006). Elaboración de instrumentos de medida de las actitudes y opiniones del profesorado universitario hacia la ética profesional docente y su papel como transmisor de valores. RELIEVE, v. 12, n. 1, p. 129-149. www.uv.es/RELIEVE/v12n1/RELIEVEv12n1_8.htm

preliminares. Teniendo en cuenta al introducir los datos, el sentido de los ítems, que en algunos casos era inverso, de tal manera que en la matriz resultante todas las respuestas seguían la misma dirección, es decir, la valoración más positiva coincidía con el cinco de la escala de respuestas, y la más negativa con el uno.

\subsection{Cuestionario sobre conocimientos,} actitudes, opiniones y expectativas hacia la ética profesional docente en la enseñanza superior.

El primer paso fue realizar un análisis de fiabilidad mediante la prueba Alfa de Cronbach, obteniéndose una fiabilidad inicial de 0'91, muy elevada, aspecto que podía resultar esperable en tanto en cuanto se trataba de un cuestionario muy extenso (114 elementos). La depuración consistió en la eliminación de aquellos ítems con correlaciones ne- gativas con el test o positivas, pero por debajo de 0 ' 40 .

Como resultado, este primer cuestionario quedó conformado con 30 ítems, sin casi pérdida de fiabilidad, que seguía prácticamente en 0'91.

Con estos elementos se procedió a la realización de un análisis factorial de componentes principales con rotación oblicua, puesto que no había motivos para pensar en la independencia de los factores, resultando un total de 8 factores que explicaban el 71 ' $33 \%$ de la varianza. Sin embargo, al contar ya, únicamente con 30 elementos, por el principio de parsimonia, decidimos forzar la solución a 4 factores.

El resultado mostró una varianza explicada del 54'28\%, y la composición de los factores pasa a describirse a continuación:

Figura 2. Composición y denominación de factores

\begin{tabular}{|c|l|c|c|}
\hline FACTOR & \multicolumn{1}{|c|}{ DENOMINACIÓN } & $\begin{array}{c}\text { VARIANZA } \\
\text { EXPLICADA }\end{array}$ & \multicolumn{1}{c|}{ ÍTEMS } \\
\hline 1 & $\begin{array}{l}\text { Respeto al alumnado y responsabilidad } \\
\text { docente }\end{array}$ & 30 '68 & $2,4,6,7,10,11,22,23,25,26$ \\
\hline 2 & Honestidad y compromiso ciudadano & $10^{\prime} 66$ & $1,3,9,24,27,28,29,30$ \\
\hline 3 & Formación ética & $7 ' 58$ & $5,16,17,18,19,20,21$ \\
\hline 4 & $\begin{array}{l}\text { Confianza en el alumnado y satisfacción } \\
\text { profesional }\end{array}$ & 5 '37 & $8,12,13,14,15$ \\
\hline
\end{tabular}

FACTOR 1. Respeto al alumnado y responsabilidad docente.- Este factor agrupa un conjunto de ítems que hace referencia a algunas competencias éticas, necesarias para el ejercicio de la docencia, como es el respeto a los alumnos/as y asumir la enorme responsabilidad que supone el ejercicio de la docencia universitaria. Trata de recoger la posición del profesorado respecto a su formación y actualización permanente, la disponibilidad a compartir conocimiento con los compañeros/as de profesión, el esfuerzo por comunicar sus conocimientos a sus alumnos/as, el autoconocimiento que posee de sus límites y su capacidad de autocrítica como. Básica- mente, agruparía competencias que, como dijimos al principio caerían dentro de lo que hemos denominado competencias éticas: las relacionadas principalmente con su tarea como transmisor de conocimientos y las relaciones con sus alumnos desde el respeto, fundamentalmente.

FACTOR 2. Honestidad y compromiso ciudadano.- También este factor está relacionado con las competencias éticas, pero, sobretodo, con su capacidad de compromiso en el ejercicio de su profesión y honestidad en sus relaciones con el alumnado, por una parte, $y$, por otra, con las actitudes del profesorado hacia su papel como formador de 
García, R., Ferrández, R, Sales, Mª A. y Moliner, Ma . O. (2006). Elaboración de instrumentos de medida de las actitudes y opiniones del profesorado universitario hacia la ética profesional docente y su papel como transmisor de valores. RELIEVE, v. 12, n. 1, p. 129-149. www.uv.es/RELIEVE/v12n1/RELIEVEv12n1_8.htm

buenos profesionales y buenos ciudadanos. Se intenta conocer el grado de importancia e implicación que el profesorado universitario concede a su papel en la formación del alumnado para que adquieran compromiso con la sociedad y con el ejercicio futuro de su profesión.

FACTOR 3. Formación ética.- Este factor recoge la percepción del profesorado sobre su capacidad para ser transmisor de valores éticos. Por ello, los ítems hacen referencia a la necesidad o no de formación en este ámbito. Lo que responde este factor es si, a juicio del profesorado, es necesario un plan de formación en la dimensión ética de la docencia; primero, como profesores y después como transmisores de estos valores éticos al alumnado.

FACTOR 4. Confianza en el alumnado y satisfacción profesional.- Este factor aglutina sólo 5 ítems y sólo explica el $5.7 \%$ de la varianza. En él se incluyen dos aspectos relacionados: el aprecio y valoración tanto de su alumnado como de su profesión. Confían en sí mismos y en sus alumnos para desarrollar su profesión. Por lo que podremos deducir su grado de satisfacción en el desempeño de la misma.

\subsection{Cuestionario sobre valores que promueve la Universidad}

Realizado el análisis de fiabilidad mediante la prueba Alfa de Cronbach, se obtuvo una fiabilidad inicial de 0 ' 88 , que se consideró, al igual que en el caso anterior, muy elevada. El cuestionario piloto constaba de 30 ítems. No obstante, al realizar la depuración mediante la eliminación de aquellos ítems con correlaciones negativas con la puntuación total en el cuestionario, o positivas pero por debajo de 0’30, la fiabilidad final, aumentó cerca de dos décimas, quedando en torno a 0'90, con un total de 24 ítems.

El análisis factorial, realizado con el fin de analizar la estructura interna del cuestionario resultante, mediante el método de Componentes principales y rotación oblicua, debido a la no asunción de independencia de los factores, nos dio, como resultado una solución con 6 factores que explicaban el $64^{\prime} 59 \%$ de la varianza. Al igual que en el análisis del cuestionario anterior se decidió forzar la solución a únicamente cuatro factores, resultando un modelo que explicaba el 54 ' $41 \%$ de la varianza, y cuya descripción se realiza a continuación.

Figura 3. Composición y denominación de factores

\begin{tabular}{|c|l|c|c|}
\hline FACTOR & \multicolumn{1}{|c|}{ DENOMINACIÓN } & $\begin{array}{c}\text { VARIANZA } \\
\text { EXPLICADA }\end{array}$ & ÍTEMS \\
\hline 1 & Enseñanza de valores en la universidad & $31^{\prime} 77$ & $1,2,3,4,5,7,9,11,18$ \\
\hline 2 & La ética profesional como asignatura & $9 ' 39$ & $10,19,20,21,22,23$ \\
\hline 3 & $\begin{array}{l}\text { Responsabilidad del profesor y del curri- } \\
\text { culum en la formación ético-profesional }\end{array}$ & 7 '16 & $6,8,14,17,24$ \\
\hline 4 & Residual & 6 '10 & $16,20,21$ \\
\hline
\end{tabular}

FACTOR 1. Enseñanza de valores en la universidad.- Este factor explica el 31,77\% de la varianza y agrupa a 9 de los 24 ítems. Detecta las actitudes del profesorado hacia dos apartados muy relacionados: por una parte, el papel que le conceden a la institución universitaria, a lo que sería la cultura universitaria, en la transmisión de valores, tanto democráticos como profesionales. Es decir, ¿es función de la universidad enseñar valores o esto ha de hacerse antes de que los alumnos acudan a ella? Por otra parte, trata de recoger la percepción del profesorado acerca de su propio papel como transmisor de valores. Se trata de conocer las respuestas 
García, R., Ferrández, R, Sales, Mª A. y Moliner, Mª O. (2006). Elaboración de instrumentos de medida de las actitudes y opiniones del profesorado universitario hacia la ética profesional docente y su papel como transmisor de valores. RELIEVE, v. 12, n. 1, p. 129-149. www.uv.es/RELIEVE/v12n1/RELIEVEv12n1_8.htm

del profesorado a cuestiones como: ¿enseñar valores es mi competencia? Si lo es, ¿qué tipo de valores? ¿éticos?, ¿profesionales?, etc.

FACTOR 2. La ética profesional como asignatura.- Una forma de eludir quizás la responsabilidad de enseñar explícitamente valores éticos al alumnado podría ser proponer la incorporación obligatoria de una asignatura que tuviera este cometido: ética de las profesiones, que tendría un título diferente, en función de la profesión concreta a la que se aplicara. Por esta razón, se plantea este factor, que trata de darnos a conocer la conveniencia o no de una materia de ética profesional, a juicio del profesorado. También nos permite conocer si esta materia ha de extenderse a todas las titulaciones o sólo aquellas de carácter social o humanístico.

FACTOR 3. Responsabilidad del profesorado y del currículo en la formación éticoprofesional.- Este factor, que explica el 7,16 de la varianza y aglutina cinco ítems, hace referencia a la responsabilidad del profesorado como modelo ético, educador de valores éticos profesionales y educador de ciudadanos responsables con su comunidad. También contempla la responsabilidad de los diseñadores de los planes de estudios para insertar en la formación superior, en cualquier titulación, y de forma transversal, el compromiso ético de los futuros profesionales.

FACTOR 4. Residual.- Está compuesto por tres ítems: uno hace referencia a la formación del profesorado para transmitir valores éticos y los otros dos a la ética profesional como asignatura.

\subsection{Cuestionario sobre valores del pro- fesorado}

La intención de este último análisis no era la eliminación de elementos, puesto que los valores incluidos en el mismo debían mantenerse por motivos de la investigación, sino en el análisis previo de la fiabilidad y estruc- tura del instrumento utilizado, con el fin de asegurar las garantías técnicas necesarias para la validez de los resultados de la investigación.

Así, en cuanto a la fiabilidad, se obtuvo un alfa de 0 '73, resultado aceptable.

\section{CONCLUSIONES}

Los instrumentos descritos han supuesto un gran esfuerzo de clarificación y concreción conceptual, así como un amplio espectro de cuestiones entorno al tema de estudio. La investigación para la que fueron diseñados y validados está actualmente en curso y consideramos que la información que están proporcionando estos instrumentos es compleja, relevante y con implicaciones pedagógicas importantes de cara a la formación y papel del profesorado universitario en el contexto del Espacio Europeo de Educación Superior. De hecho, en los últimos años, no es extraño escuchar voces que reivindican la necesidad de incluir la formación ética del alumnado universitario, así como el reconocimiento de la función educativa del profesorado universitario, sin embargo, son pocos los estudios e investigaciones que constaten que, efectivamente, el profesorado universitario se ve así mismo, no sólo como transmisor de conocimientos, sino también como educador $\mathrm{y}$, por tanto, también transmisor de valores relacionados con la ciencia, el saber, el saber hacer, el saber vivir y el saber ser (Martínez y Esteban, 2005). Esta es una de las pretensiones de nuestro trabajo, ya que la formación ética profesional lleva consigo la necesaria formación de todo el profesorado universitario, que si bien llega a recibir formación inicial en otros ámbitos (nuevas tecnologías, planificación docente, sistemas de evaluación, tutorías...), tiene una gran laguna formativa en la enseñanza de actitudes y valores. Para muchos profesores las actitudes y valores no deben explicitarse, sino que se aprenden y enseñan de una manera espontánea y no programada. Sin embargo, consideramos que es mucho más pedagógico y efectivo hacer ex- 
García, R., Ferrández, R, Sales, Mª A. y Moliner, Mª O. (2006). Elaboración de instrumentos de medida de las actitudes y opiniones del profesorado universitario hacia la ética profesional docente y su papel como transmisor de valores. RELIEVE, v. 12, n. 1, p. 129-149. www.uv.es/RELIEVE/v12n1/RELIEVEv12n1_8.htm

plícita su formación, justamente para evitar la manipulación y el adoctrinamiento, para favorecer el aprendizaje responsable y consciente de la dimensión ética de toda profesión. De lo contrario, dejamos a la voluntad y criterio de cada profesor la conveniencia de abordar este aspecto fundamental o de no hacerlo, en detrimento de una visión y formación global y comprometida de su profesión.

La consecución de esos objetivos no depende sólo del profesorado, de su formación y del reconocimiento de su dimensión más educativa dentro de su tarea docente; también depende de la cultura universitaria en la que se inserta este proyecto. Con la convergencia europea, son muchos los cambios que han de operarse para conseguir una cultura universitaria en la que el profesorado desempeñe funciones que hasta ahora no se contemplaban de forma explícita. Nuestra investigación trata de explorar y analizar, a partir de los instrumentos elaborados y validados que presentamos aquí, las actitudes de los propios docentes para favorecer una cambio positivo hacia un mayor compromiso ético con la profesión.

\section{BIBLIOGRAFÍA}

Bolívar, A. (2005) El Lugar De La Ética Profesional En La Formación Universitaria, En Rmie, Ener-Mar, Vol. 10, Núm. 24, Pp. 93123

Cobo, J. M. (2001) Ética Profesional En Ciencias Humanas $Y$ Sociales. Madrid: Huerga Fierro Editores.

Escámez, J.; Ortega, P. y Martínez, M. (2005): "Los Valores De La Educación En El Espacio Europeo De La Enseñanza Superior", En Esteban Chapapría, V. (Editor): El Espacio Europeo De Educación Superior. Valencia: Ed. Upv (Universidad Politéctica De Valencia), Pp. 165-198.

Esteban Bara, F. (2004) Excelentes Profesionales y Comprometidos Ciudadanos. Un Cambio De Mirada Desde La Universidad. Col. Aprender A Ser Desclée De Brouwer, Bilbao.
García López, R. Et Al. (2005) El Profesorado Universitario Ante La Enseñanza De Valores. Actas Del Seminario Interuniversitario De Teoría De La Educación. Valencia.

Gross, B. Romanya, T. (2004) Ser Profesor. Palabras Sobre La Docencia Universitaria. Barcelona: Octaedro/Ice-Ub, .

Hortal, A. (2002) Ética General De Las Profesiones. Bilbao, Desclée De Brouwer, S. A. Jover, G.; Fernández, C. y Ruiz, M. (2005). El Diseño de Titulaciones y programas ante la Convergencia Europea.,En Esteban Chapapría, V. (Editor): El Espacio Europeo De Educación Superior. Valencia: Ed. Upv (Universidad Politéctica De Valencia), , Pp. 27-93.

Martínez, M.; Buxarrais, M ${ }^{\mathrm{a}}$ R. y Esteban, F. (2002). Ética y Formación Universitaria. Revista Iberoamericana De Educación, $\mathrm{N}^{\mathrm{o}}$ 29, Pp. 19-43.

Martínez, M. Y Esteban, F. (2005). Una Propuesta De Formación Ciudadana Para El EEES. Revista Española De Pedagogía, 230: 63-83.

Rokeach, M. (1973) The Nature Of Human Values. New York: Free Press.

Rubio, M. J. y Varas, J. (1997). El Análisis De La Realidad, En La Intervención Social. Métodos Y Técnicas De Investigación. Madrid: Editorial Ccs.

Tójar, J.C. y Serrano, J. (2000). Ética e investigación educativa. RELIEVE, vol. $6, \mathrm{n}$. 2. Consultado en http://www.uv.es/RELIEVE/v6n2/RELIEV Ev6n2 2.htmel 5 de Mayo de 2006

Zabalza, M. (2002) La Enseñanza Universitaria. El Escenario y Sus Protagonistas. Madrid: Narcea.

Zabalza, M. (2003) Competencias Docentes Del Profesorado Universitario. Calidad Y Desarrollo Profesional. Madrid: Narcea.

\section{NOTAS}

[1] Los siguientes miembros :Juan Escámez, Gonzalo Jover, Fernando Gil, Auxiliadora Sales, David Reyero, Reina Ferrández, Odet Moliner, Belen Zayas pertenecen al equipo de esta investigación, dirigido por 
García, R., Ferrández, R, Sales, Mª A. y Moliner, Mª O. (2006). Elaboración de instrumentos de medida de las actitudes y opiniones del profesorado universitario hacia la ética profesional docente y su papel como transmisor de valores. RELIEVE, v. 12, n. 1, p. 129-149. www.uv.es/RELIEVE/v12n1/RELIEVEv12n1_8.htm

Rafaela García López, subvencionada por la Consellería de Educación, Cultura y Deportes de la Generalitat Valenciana.
[2] Las respuestas que se dieron en los Grupos de Discusión fueron el fundamento para realizar gran parte de los instrumentos de esta investigación.

\title{
ANEXO 1 CUESTIONARIO DEFINITIVO
}

\section{CUESTIONARIO DE ACTITUDES Y OPINIONES DEL PROFESORADO UNIVERSITARIO SOBRE LA ÉTICA PROFESIONAL DOCENTE}

\begin{abstract}
Estimado compañero/a nos dirigimos a usted en demanda de cooperación para llevar a cabo una investigación sobre las actitudes del profesorado universitario hacia la ética profesional docente y hacia la formación de valores en la universidad. Esta investigación está siendo desarrollada por un equipo de profesores y profesoras de la Universidad de Valencia, de la Universidad Complutense de Madrid y de la Universidad Jaume I de Castellón. El proyecto ha sido avalado por la Consellería de Cultura, Educación y Deporte de la Generalitat Valenciana, dentro del Plan I+D de 2004

Uno de los objetivos que perseguimos es conocer la valoración que el profesorado universitario da a la ética profesional docente y a su papel como transmisor de valores. Pensamos que de las respuestas obtenidas pueden influir en futuros planes de formación del profesorado universitario.

Su colaboración es imprescindible para llevarla a cabo y le agradecemos su participación en el mismo.

Muchas gracias por su colaboración
\end{abstract}

DATOS DEL PROFESOR/A:

Universidad:

$\square$ Universidad de Valencia

$\square$ Universidad Jaime I de Castellón

$\square$ Universidad Complutense de Madrid

Facultad o Escuela:

Sexo:

$\square$ Hombre

$\square$ Mujer

Edad:
$\square$ Menos de 35
$\square$ 36-40
$\square$ 41-45
$\square$ 46-50
$\square$ 51-55
$\square$ 56-60
$\square$ Más de 60 años

Años de experiencia docente en la universidad:
$\square$ 0-5
$\square$ 6-10
$\square$ 11-15
$\square$ 16-20
$\square$ 21-25
$\square$ 26-30
$\square$ Más de 30 años

Titulación académica:
$\square$ Licenciado, arquitecto o equivalente
$\square$ Doctor

$\square$ Otros 
García, R., Ferrández, R, Sales, Mª A. y Moliner, Ma . O. (2006). Elaboración de instrumentos de medida de las actitudes y opiniones del profesorado universitario hacia la ética profesional docente y su papel como transmisor de valores. RELIEVE, v. 12, n. 1, p. 129-149. www.uv.es/RELIEVE/v12n1/RELIEVEv12n1_8.htm

Situación administrativa-profesional:

\section{$\square$ Ayudante}

$\square$ Contratado doctor

$\square$ Profesor/a T.E.U.

$\square$ Profesor/a T.U.

$\square$ Catedrático/a de E.U.

$\square$ Catedrático/a Universidad

$\square$ Otro

1. CUESTIONARIO SOBRE CONOCIMIENTOS, ACTITUDES, OPINIONES Y EXPECTATIVAS HACIA LA ÉTICA PROFESIONAL DOCENTE EN LA ENSEÑANZA SUPERIOR

1. Indique el grado de acuerdo de 1 (poco) a 5 (mucho) con las siguientes afirmaciones:

\begin{tabular}{|c|c|c|c|c|c|}
\hline $\begin{array}{l}\text { a. La ética profesional docente consiste en reflexionar constantemente sobre la } \\
\text { propia práctica para mejorarla }\end{array}$ & 1 & 2 & 3 & 4 & 5 \\
\hline $\begin{array}{l}\text { b. La ética profesional docente supone aceptar un código ético consensuado } \\
\text { como pautas a seguir }\end{array}$ & 1 & 2 & 3 & 4 & 5 \\
\hline $\begin{array}{l}\text { c. La ética profesional docente exige justificar las propias actuaciones profe- } \\
\text { sionales }\end{array}$ & 1 & 2 & 3 & 4 & 5 \\
\hline $\begin{array}{l}\text { d. La ética profesional docente hace referencia a las normas de comportamien- } \\
\text { to correcto que se deben cumplir en el ejercicio de la profesión docente }\end{array}$ & 1 & 2 & 3 & 4 & 5 \\
\hline $\begin{array}{l}\text { e. La ética profesional resuelve los dilemas a los que se enfrentan los docentes } \\
\text { en su tarea diaria }\end{array}$ & 1 & 2 & 3 & 4 & 5 \\
\hline $\begin{array}{l}\text { f. La ética profesional docente hace referencia al conjunto de valores y princi- } \\
\text { pios morales en los que se sustenta el ejercicio profesional y orientan cómo } \\
\text { deben desarrollarse las tareas del docente }\end{array}$ & 1 & 2 & 3 & 4 & 5 \\
\hline $\begin{array}{l}\text { h. La ética profesional docente supone la asunción de valores que implican } \\
\text { normas de comportamiento profesional y modelos de actuación en el ejercicio } \\
\text { de la profesión }\end{array}$ & 1 & 2 & 3 & 4 & 5 \\
\hline $\begin{array}{l}\text { i. La ética profesional también supone evaluar la propia actuación y tomar } \\
\text { decisiones }\end{array}$ & 1 & 2 & 3 & 4 & 5 \\
\hline $\begin{array}{l}\text { j. La ética profesional docente también supone tomar conciencia de la tarea } \\
\text { docente }\end{array}$ & 1 & 2 & 3 & 4 & 5 \\
\hline
\end{tabular}

2. Marque el grado de importancia que, a su juicio, hoy se le concede por parte de los docentes universitarios, a la ética profesional docente ${ }^{[1]}$.

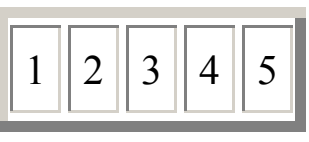

3. Y ¿qué grado de importancia le concede usted para su propio ejercicio profesional?

$$
\begin{array}{|l|l|l|l|l|}
\hline 1 & 2 & 3 & 4 & 5 \\
\hline
\end{array}
$$

4. ¿Considera que el profesorado universitario debería disponer de un código ético para ejercer su docencia?
SI $\square$
NO $\square$
$\mathrm{NS} / \mathrm{NC}$ 
García, R., Ferrández, R, Sales, Mª A. y Moliner, Ma . O. (2006). Elaboración de instrumentos de medida de las actitudes y opiniones del profesorado universitario hacia la ética profesional docente y su papel como transmisor de valores. RELIEVE, v. 12, n. 1, p. 129-149. www.uv.es/RELIEVE/v12n1/RELIEVEv12n1_8.htm

5. En caso afirmativo, marque lo que, a su juicio, debería regular:

Las relaciones con los alumnos/as

Las relaciones con los compañeros/as

Las relaciones con la institución universitaria

Las relaciones con la sociedad

La formación permanente (Relación de uno mismo como profesional interesado en el desarrollo y actualización en el área de conocimiento a la que pertenece)

Las relaciones con la investigación

Relación con el secreto profesional

Otras Especificar

6. ¿Considera que sólo las profesiones que tengan una dimensión social exigen una deontología?

$$
\text { SI } \square \quad \mathrm{NO} \square \quad \mathrm{NS} / \mathrm{NC}
$$

7. ¿Está de acuerdo con que la práctica docente, además de ser una cuestión técnico-instrumental, también es una cuestión moral?

$$
\text { SI } \square \quad \text { NO } \square
$$

8. Ordene de menor (1) a mayor (5) importancia las competencias que caracterizan a un buen docente universitario

Competencias técnico-organizativas (metodologías)

Competencias sociales

Competencias éticas

Competencias afectivo-emocionales

Competencias de conocimiento (Teórico-científicas)

\section{CUESTIONARIO DE ACTITUDES}

Con respecto a cada ítem le rogamos que valore y marque con una $\mathbf{X}$, según la siguiente escala de valoración:

1. Muy en desacuerdo

2. En desacuerdo

3. Indeciso

4. De acuerdo

5. Muy de acuerdo 
García, R., Ferrández, R, Sales, Mª A. y Moliner, Ma . O. (2006). Elaboración de instrumentos de medida de las actitudes y opiniones del profesorado universitario hacia la ética profesional docente y su papel como transmisor de valores. RELIEVE, v. 12, n. 1, p. 129-149. www.uv.es/RELIEVE/v12n1/RELIEVEv12n1_8.htm

1. Me preocupo de conocer las inquietudes de mis alumnos preparando nuevos temas para motivarlos.

2. Estoy dispuesto/a a dedicar parte de mi tiempo a seguir formándome para mejorar mi docencia.

3. Acepto el riesgo de equivocarme con tal de mejorar mi actividad docente.

4. Compartir mis conocimientos con mis compañeros me enriquece personal y profesionalmente.

5. Necesito ponerme en lugar de mis alumnos/as para comprender sus necesidades

6. De nada sirven mis conocimientos si no logro comunicarlos bien a los alumnos/as.

7. Puedo resolver algunos problemas escuchando a mis alumnos.

8. El docente universitario debe adaptar sus conocimientos a las características del alumnado.

9. Cumplo los compromisos que establezco con mis alumnos/as.

10. Para no cometer errores en el ejercicio de la docencia debo ser consciente de los límites de mis conocimientos y habilidades.

11. Intento no engañar a mis alumnos sobre mis conocimientos.

12. Confío en que todos mis alumnos pueden aprender.

13. Estoy satisfecho/a con mi profesión.

14. Es un logro hacer profesionalmente lo que más me gusta.

15. Para ejercer bien la docencia es importante dar un poco de uno mismo/a.

16. No es necesaria la formación ética para ser un buen docente universitario.

17. Estoy dispuesto a formarme en cuestiones éticas relacionadas con la docencia.

18. Deben existir cursos obligatorios para todo el profesorado sobre ética profesional docente.

19. La formación ética aumenta el prestigio de mi profesión.

20. No necesito formación ética para el ejercicio responsable de mi profesión.

21. La universidad debe obligar a los profesores noveles a cursar unas horas de formación sobre la ética docente.

22. Para ser un buen profesor he de ser crítico conmigo mismo.

23. Un profesor debe conocer y respetar los derechos de los alumnos/as.

24. Procuro no denegar beneficios a los alumnos que trabajan, y busco con ellos soluciones para su aprendizaje.

25. Me considero en el deber de orientar a mis alumnos, estimularlos para el aprendizaje y enseñarles todo lo que pueda de mi materia.

26. No acepto a aquellos compañeros o compañeras que explotan a sus alumnos.

27. Intento que mis alumnos/as reflexionen sobre el papel profesional que desempeñarán en la sociedad.

28. Formo a mis alumnos para que, además de que sean buenos profesionales, trabajen por el bien común.

29. Colaboro en la mejora de mi Facultad o Escuela.

30. Intento transmitir a mis alumnos el sentido de pertenencia a la comunidad universitaria.

\begin{tabular}{|c|c|c|c|c|}
\hline 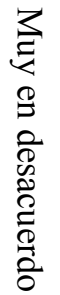 & 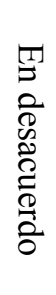 & 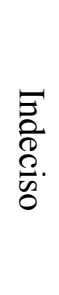 & 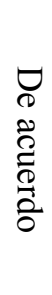 & $\begin{array}{l}3 \\
\text { द्र } \\
\text { के } \\
\text { है } \\
0 \\
0 \\
0 \\
0\end{array}$ \\
\hline 1 & 2 & 3 & 4 & 5 \\
\hline 1 & 2 & 3 & 4 & 5 \\
\hline 1 & 2 & 3 & 4 & 5 \\
\hline 1 & 2 & 3 & 4 & 5 \\
\hline 1 & 2 & 3 & 4 & 5 \\
\hline 1 & 2 & 3 & 4 & 5 \\
\hline 1 & 2 & 3 & 4 & 5 \\
\hline 1 & 2 & 3 & 4 & 5 \\
\hline 1 & 2 & 3 & 4 & 5 \\
\hline 1 & 2 & 3 & 4 & 5 \\
\hline 1 & 2 & 3 & 4 & 5 \\
\hline 1 & 2 & 3 & 4 & 5 \\
\hline 1 & 2 & 3 & 4 & 5 \\
\hline 1 & 2 & 3 & 4 & 5 \\
\hline 1 & 2 & 3 & 4 & 5 \\
\hline 1 & 2 & 3 & 4 & 5- \\
\hline 1 & 2 & 3 & 4 & 5 \\
\hline 1 & 2 & 3 & 4 & 5 \\
\hline 1 & 2 & 3 & 4 & 5 \\
\hline 1 & 2 & 3 & 4 & $5-$ \\
\hline 1 & 2 & 3 & 4 & 5 \\
\hline 1 & 2 & 3 & 4 & 5 \\
\hline 1 & 2 & 3 & 4 & 5 \\
\hline 1 & 2 & 3 & 4 & 5 \\
\hline 1 & 2 & 3 & 4 & 5 \\
\hline 1 & 2 & 3 & 4 & 5 \\
\hline 1 & 2 & 3 & 4 & 5 \\
\hline 1 & 2 & 3 & 4 & 5 \\
\hline 1 & 2 & 3 & 4 & 5 \\
\hline 1 & 2 & 3 & 4 & 5 \\
\hline
\end{tabular}


García, R., Ferrández, R, Sales, Mª A. y Moliner, Mª O. (2006). Elaboración de instrumentos de medida de las actitudes y opiniones del profesorado universitario hacia la ética profesional docente y su papel como transmisor de valores. RELIEVE, v. 12, n. 1, p. 129-149. www.uv.es/RELIEVE/v12n1/RELIEVEv12n1_8.htm

\section{ANEXO 2}

\section{CUESTIONARIO SOBRE VALORES QUE PROMUEVE LA UNIVERSIDAD}

1. Indique ¿en qué grado cree que la Universidad, como institución, debe desarrollar los valores de sus alumnos/as?

$$
\text { Mucho } \square \quad \text { Poco } \square \quad \text { Nada } \square
$$

2. Marque el tipo de valores que, prioritariamente, debe desarrollar la universidad ${ }^{[2]}$ :

\begin{tabular}{|l|l|l|l|l|l|l|}
\hline 2.1. Valores democráticos & 1 & 2 & 3 & 4 & 5 \\
\hline 2.2. Valores éticos & 1 & 2 & 3 & 4 & 5 \\
\hline 2.3. Valores profesionales & 1 & 2 & 3 & 4 & 5 \\
\hline 2.4. Valores cívicos & 1 & 2 & 3 & 4 & 5 \\
\hline 2.5. Valores de conocimiento & 1 & 2 & 3 & 4 & 5 \\
\hline 2.6. Otros. Especificar: & 1 & 2 & 3 & 4 & 5 \\
\hline
\end{tabular}

3. ¿Es la universidad un espacio adecuado para desarrollar valores de ciudadanía?

SI $\square \quad$ NO $\square \quad$ NS/NC $\square$

4. En caso de responder afirmativamente a pregunta anterior, señale el grado - de menos (1) a más (5)- en que los siguientes factores dificultan la enseñanza de valores:

\begin{tabular}{|l|l|l|l|l|l|}
\hline 4.1. La organización de la docencia & 1 & 2 & 3 & 4 & 5 \\
\hline 4.2. La extensión de los contenidos de las disciplinas & 1 & 2 & 3 & 4 & 5 \\
\hline 4.3. Problemas del carácter de la materia & 1 & 2 & 3 & 4 & 5 \\
\hline 4.4. Desinterés y apatía del alumnado & 1 & 2 & 3 & 4 & 5 \\
\hline 4.5. Desinterés del profesorado & 1 & 2 & 3 & 4 & 5 \\
\hline 4.6. Falta de preparación del profesorado & 1 & 2 & 3 & 4 & 5 \\
\hline 4.7. Desconocimiento de metodología docente & 1 & 2 & 3 & 4 & 5 \\
\hline 4.8. Otros problemas. Especificar: & 1 & 2 & 3 & 4 & 5 \\
\hline
\end{tabular}

5. Exponga los cinco valores básicos que la universidad debería de promover en su alumnado.

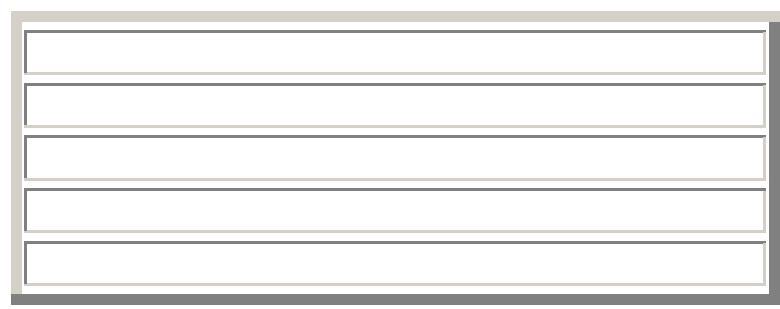


García, R., Ferrández, R, Sales, Mª A. y Moliner, Mª O. (2006). Elaboración de instrumentos de medida de las actitudes y opiniones del profesorado universitario hacia la ética profesional docente y su papel como transmisor de valores. RELIEVE, v. 12, n. 1, p. 129-149. www.uv.es/RELIEVE/v12n1/RELIEVEv12n1_8.htm

6. Exponga los cinco valores básicos que la universidad debería de promover en su profesorado.

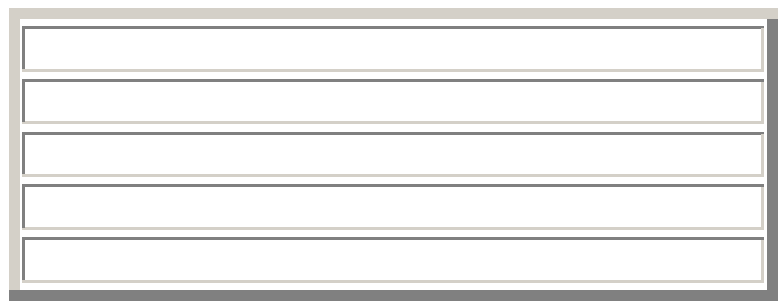

8. ¿Debería existir una materia de ética profesional para el alumnado en todas las titulaciones?

$$
\text { SI } \square \quad \mathrm{NO} \square \quad \mathrm{NS} / \mathrm{NC} \square
$$

9. ¿Qué contenidos debería tener esa materia?

\section{CUESTIONARIO DE ACTITUDES HACIA VALORES}

Con respecto a cada ítem le rogamos que valore y marque con una $\mathbf{X}$, según la siguiente escala de valoración:

1. Muy en desacuerdo

2. En desacuerdo

3. Indeciso

4. De acuerdo

5. Muy de acuerdo 
García, R., Ferrández, R, Sales, Ma . A. y Moliner, Mª O. (2006). Elaboración de instrumentos de medida de las actitudes y opiniones del profesorado universitario hacia la ética profesional docente y su papel como transmisor de valores. RELIEVE, v. 12, n. 1, p. 129-149. www.uv.es/RELIEVE/v12n1/RELIEVEv12n1_8.htm

\begin{tabular}{|c|c|c|c|c|c|}
\hline & 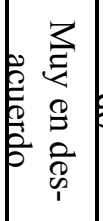 & 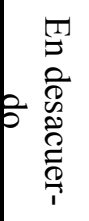 & 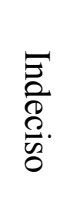 & $\begin{array}{l}\underset{0}{0} \\
\stackrel{0}{0} \\
\stackrel{0}{0} \\
\stackrel{0}{0}\end{array}$ & 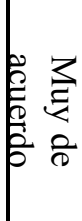 \\
\hline 1. Considero que no es función de la Universidad enseñar valores a sus alumnos/as. & 1 & 2 & 3 & 4 & $5-$ \\
\hline $\begin{array}{l}\text { 2. La universidad tiene la obligación de transmitir los valores democráticos a sus } \\
\text { alumnos/as. }\end{array}$ & 1 & 2 & 3 & 4 & 5 \\
\hline 3. La universidad sólo debe enseñar valores profesionales. & 1 & 2 & 3 & 4 & $5-$ \\
\hline 4. No es mi función influir en la escala de valores de los alumnos/as. & 1 & 2 & 3 & 4 & $5-$ \\
\hline 5. Cada persona tiene sus propios valores y no me corresponde cuestionarlos. & 1 & 2 & 3 & 4 & $5-$ \\
\hline $\begin{array}{l}\text { 6. El profesorado tiene la obligación de transmitir a sus alumnos los principios } \\
\text { éticos de la profesión para las que se están formando. }\end{array}$ & 1 & 2 & 3 & 4 & 5 \\
\hline 7. Enseñar valores es peligroso porque se cae en el adoctrinamiento. & 1 & 2 & 3 & 4 & $5-$ \\
\hline 8. Como profesor debo ser modelo ético para mis alumnos/as. & 1 & 2 & 3 & 4 & 5 \\
\hline 9. No es mi competencia enseñar valores éticos a mis alumnos/as. & 1 & 2 & 3 & 4 & $5-$ \\
\hline $\begin{array}{l}\text { 10. No necesito una preparación específica para transmitir valores éticos a mis } \\
\text { alumnos/as. }\end{array}$ & 1 & 2 & 3 & 4 & $5-$ \\
\hline 11. Los valores profesionales se aprenden sólo cuando se ejercita la profesión. & 1 & 2 & 3 & 4 & $5-$ \\
\hline 12. Mi competencia profesional nada tiene que ver con mi competencia ética. & 1 & 2 & 3 & 4 & $5-$ \\
\hline 13. No se pueden formar buenos profesionales que sean malos ciudadanos. & 1 & 2 & 3 & 4 & 5 \\
\hline 14. También es responsabilidad del profesor formar buenos ciudadanos. & 1 & 2 & 3 & 4 & 5 \\
\hline 15. Los alumnos no aceptan los valores que yo trato de enseñarles. & 1 & 2 & 3 & 4 & 5 \\
\hline 16. No conozco una adecuada metodología para enseñar valores & 1 & 2 & 3 & 4 & 5 \\
\hline 17. Con una buena metodología se puede educar éticamente sin adoctrinar & 1 & 2 & 3 & 4 & 5 \\
\hline $\begin{array}{l}\text { 18. No puedo imponer valores a mis alumnos, pero sí ayudarles a clarificar su esca- } \\
\text { la de valores }\end{array}$ & 1 & 2 & 3 & 4 & 5 \\
\hline 19. Considero irrelevante una disciplina de ética profesional. & 1 & 2 & 3 & 4 & 5- \\
\hline 20. Una asignatura sobre ética profesional es necesaria en todas las Titulaciones & 1 & 2 & 3 & 4 & $5-$ \\
\hline $\begin{array}{l}\text { 21. La asignatura sobre ética profesional sólo es necesaria en las Titulaciones so- } \\
\text { ciales y humanas, no en las técnicas }\end{array}$ & 1 & 2 & 3 & 4 & $5-$ \\
\hline 22. No es recomendable una asignatura sobre ética profesional. & 1 & 2 & 3 & 4 & $5-$ \\
\hline $\begin{array}{l}\text { 23. Es conveniente una asignatura sobre ética profesional en forma de créditos } \\
\text { prácticos, con casos reales y simulaciones }\end{array}$ & 1 & 2 & 3 & 4 & 5 \\
\hline $\begin{array}{l}\text { 24. El componente ético profesional debe impregnar todas las asignaturas, no con- } \\
\text { centrarse en una disciplina. }\end{array}$ & 1 & 2 & 3 & 4 & 5 \\
\hline
\end{tabular}


García, R., Ferrández, R, Sales, Mª A. y Moliner, Ma . O. (2006). Elaboración de instrumentos de medida de las actitudes y opiniones del profesorado universitario hacia la ética profesional docente y su papel como transmisor de valores. RELIEVE, v. 12, n. 1, p. 129-149. www.uv.es/RELIEVE/v12n1/RELIEVEv12n1_8.htm

\section{ANEXO $\cdot 3$}

\section{CUESTIONARIO SOBRE VALORES DEL PROFESORADO}

Puntúe de 1 a 7 los valores que le presentamos a continuación, de acuerdo con la importancia que tienen para usted en su ejercicio profesional, sabiendo que 1 es el mínimo de importancia y 7 el máximo:

\begin{tabular}{|c|c|c|c|c|c|c|c|}
\hline 1. Autoestima ( consideración negativa o positiva hacia sí mismo/a) & 1 & 2 & 3 & 4 & 5 & 6 & 7 \\
\hline $\begin{array}{l}\text { 2. Competencia ( Seguridad en sus capacidades para realizar las tareas o } \\
\text { trabajos) }\end{array}$ & 1 & 2 & 3 & 4 & 5 & 6 & 7 \\
\hline 3. Responsabilidad ( Estar dispuesto/a a asumir las consecuencias) & 1 & 2 & 3 & 4 & 5 & 6 & 7 \\
\hline $\begin{array}{l}\text { 4. Respeto ( Aceptación de las ideas, opiniones y gustos distintos a los su- } \\
\text { yos) }\end{array}$ & 1 & 2 & 3 & 4 & 5 & 6 & 7 \\
\hline 5. Participación ( Implicación activa en proyectos personales y sociales) & 1 & 2 & 3 & 4 & 5 & 6 & 7 \\
\hline $\begin{array}{l}\text { 6. Sentido crítico (Capacidad de contrastar distintas opiniones atendiendo a } \\
\text { las razones que las fundamentan y no a la presión de los demás). }\end{array}$ & 1 & 2 & 3 & 4 & 5 & 6 & 7 \\
\hline 7. Amistad ( Comprensión, aprecio y aceptación de los otros cercanos) & 1 & 2 & 3 & 4 & 5 & 6 & 7 \\
\hline $\begin{array}{l}\text { 8. Autonomía ( Independencia de pensar, tomar decisiones y actuar, procu- } \\
\text { rando tener dominio en la dirección de su propia vida) }\end{array}$ & 1 & 2 & 3 & 4 & 5 & 6 & 7 \\
\hline $\begin{array}{l}\text { 9. Bienestar ( Hacer las cosas que le gustan en la vida, ya sea desde la co- } \\
\text { modidad o desde lo estimulante y arriesgado) }\end{array}$ & 1 & 2 & 3 & 4 & 5 & 6 & 7 \\
\hline $\begin{array}{l}\text { 10. Convivencia (Aceptar los sentimientos, actitudes y opiniones de las } \\
\text { demás personas que conviven con usted). }\end{array}$ & 1 & 2 & 3 & 4 & 5 & 6 & 7 \\
\hline $\begin{array}{l}\text { 11. Cooperación (Ayudar a los demás o pedirles ayuda para conseguir pro- } \\
\text { pósitos comunes). }\end{array}$ & 1 & 2 & 3 & 4 & 5 & 6 & 7 \\
\hline $\begin{array}{l}\text { 12. Felicidad (Tener un sentido de realización personal, de hacer algo útil, } \\
\text { importante). }\end{array}$ & 1 & 2 & 3 & 4 & 5 & 6 & 7 \\
\hline $\begin{array}{l}\text { 13. Constancia (Saber autocontrolarse y ser capaz de acabar las tareas ini- } \\
\text { ciadas). }\end{array}$ & 1 & 2 & 3 & 4 & 5 & 6 & 7 \\
\hline 14. Sinceridad (Ser honesto/a, decir la verdad). & 1 & 2 & 3 & 4 & 5 & 6 & 7 \\
\hline 15. Libertad (Poder obrar de una manera u otra, o dejar de actuar) & 1 & 2 & 3 & 4 & 5 & 6 & 7 \\
\hline $\begin{array}{l}\text { 16. Diálogo (Búsqueda del acuerdo o deliberación a través del intercambio } \\
\text { de argumentos con otros) }\end{array}$ & 1 & 2 & 3 & 4 & 5 & 6 & 7 \\
\hline $\begin{array}{l}\text { 17. Coherencia (Mantener unidad de criterios entre las propias convicciones } \\
\text { y actuaciones). }\end{array}$ & 1 & 2 & 3 & 4 & 5 & 6 & 7 \\
\hline 18. Civismo (Compromiso con el bien público) & 1 & 2 & 3 & 4 & 5 & 6 & 7 \\
\hline $\begin{array}{l}\text { 19. Flexibilidad (Capacidad para adaptarse a diferentes contextos y situa- } \\
\text { ciones) }\end{array}$ & 1 & 2 & 3 & 4 & 5 & 6 & 7 \\
\hline 20. Reconocimiento social ( Ser valorado o reconocido socialmente) & 1 & 2 & 3 & 4 & 5 & 6 & 7 \\
\hline 21. Seguridad (Conservar el trabajo, los compañeros, el status) & 1 & 2 & 3 & 4 & 5 & 6 & 7 \\
\hline 22. Innovación ( Introducir cambios para mejorar) & 1 & 2 & 3 & 4 & 5 & 6 & 7 \\
\hline $\begin{array}{l}\text { 23. Liderazgo (Ser capaz de guiar y orientar, de tener influencia sobre los } \\
\text { demás) }\end{array}$ & 1 & 2 & 3 & 4 & 5 & 6 & 7 \\
\hline $\begin{array}{l}\text { 24. Perfectibilidad (Necesidad de mejorar, perfeccionarse y desarrollarse } \\
\text { profesionalmente) }\end{array}$ & 1 & 2 & 3 & 4 & 5 & 6 & 7 \\
\hline
\end{tabular}

[1] La valoración siempre es de menos (1) a más (5).

[2] La valoración siempre es de menos (1) a más (5). 


\section{ABOUT THE AUTHORS / SOBRE LOS AUTORES}

Rafaela García López (rafaela.garcia@uv.es). Doctora en Pedagogía, Lda. en Psicología y profesora Titular del Departamento de Teoría de la Educación de la Universidad de Valencia. Profesora Invitada en la Universidad Iberoamericana de México. Ha publicado numerosos trabajos sobre formación de actitudes, valores y educación moral y cívica. Sus líneas de investigación son: pedagogía de las actitudes y valores; educación moral y cívica, educación intercultural y ética profesional. En la actualidad dirige varias investigaciones sobre educación intercultural y ética profesional docente en el marco del nuevo Espacio Europeo de Enseñanza Superior.

$\mathbf{M}^{\mathrm{a}}$ Reina Ferrández Berrueco (ferrande@edu.uji.es). Licenciada y doctora en Filosofía y Ciencias de la Educación (sección CC. De la Educación) por la Universidad de Valencia. Profesora Contratada Doctora del Departamento de Educación de la Universitat Jaume I en el Área de Métodos de Investigación y Diagnóstico en Educación. Centra su línea de investigación en evaluación de la calidad así como en aspectos metodológicos asociados a la investigación educativa.

$\mathbf{M}^{\mathbf{a}}$ Auxiliadora Sales Ciges (asales@edu.uji.es ). Licenciada y doctora en Filosofía y Ciencias de la Educación (sección CC. de la Educación) por la Universidad de Valencia, con premios extraordinarios de Licenciatura y de Doctorado. Profesora Titular del Departamento de Educación de la Universitat Jaume I. Centra su línea de investigación en la educación intercultural e inclusiva, participando en distintos proyectos investigadores tanto de ámbito local como autonómico.

Ma Odet Moliner García (molgar@edu.uji.es). Licenciada y doctora en Filosofía y Ciencias de la Educación (sección CC. de la Educación) por la Universidad de Valencia. Profesora Contratada Doctora del Departamento de Educación de la Universidad Jaume I de Castellón, centra su línea investigadora en la formación de actitudes y valores y la educación en la diversidad. 
García, R., Ferrández, R, Sales, Mª A. y Moliner, Ma . O. (2006). Elaboración de instrumentos de medida de las actitudes y opiniones del profesorado universitario hacia la ética profesional docente y su papel como transmisor de valores. RELIEVE, v. 12, n. 1, p. 129-149. www.uv.es/RELIEVE/v12n1/RELIEVEv12n1_8.htm

\section{ARTICLE RECORD / FICHA DEL ARTÍCULO}

\begin{tabular}{|c|c|}
\hline $\begin{array}{l}\text { Reference / } \\
\text { Referencia }\end{array}$ & $\begin{array}{l}\text { García, Rafaela, Ferrández, } \mathrm{M}^{\mathrm{a}} \text { Reina, Sales, } \mathrm{M}^{\mathrm{a}} \text { Auxiliadora, Moliner, } \mathrm{M}^{\mathrm{a}} \text { Odet (2006). Elaboración } \\
\text { de instrumentos de medida de las actitudes y opiniones del profesorado universitario hacia la ética } \\
\text { profesional docente y su papel como transmisor de valores. } \\
\text { httpLIEVE, v. } 12, \text { n. } 2 \text {. } \\
\text { hww.uv.es/RELIEVE/v12n1/RELIEVEv12n1 8.htm. Consultado en (poner fecha). }\end{array}$ \\
\hline Title / Título & $\begin{array}{l}\text { Elaboración de instrumentos de medida de las actitudes y opiniones del profesorado universitario } \\
\text { hacia la ética profesional docente y su papel como transmisor de valores. [Elaboration of measure- } \\
\text { ment instruments for attitudes and opinions from university academic personnel towards teaching } \\
\text { professional Ethics and its role as values transmitter] }\end{array}$ \\
\hline $\begin{array}{l}\text { Authors / } \\
\text { Autores }\end{array}$ & Rafaela García, M ${ }^{\mathrm{a}}$ Reina Ferrández, M $^{\mathrm{a}}$ Auxiliadora, Sales y Ma Odet Moliner \\
\hline $\begin{array}{l}\text { Review / } \\
\text { Revista }\end{array}$ & Revista ELectrónica de Investigación y EValuación Educativa (RELIEVE), v. 12, n. 1 \\
\hline ISSN & $1134-4032$ \\
\hline $\begin{array}{l}\text { Publication } \\
\text { date / Fecha } \\
\text { de publicación }\end{array}$ & $\begin{array}{l}2006 \text { (Reception Date: } 2003 \text { March 09; Approval Date: } 2006 \text { June 20; Publication Date: } 2006 \text { Ju- } \\
\text { ne 22) }\end{array}$ \\
\hline $\begin{array}{l}\text { Abstract / } \\
\text { Resumen }\end{array}$ & $\begin{array}{l}\text { From the research study - a compared analysis of the university academic personnel's attitudes and } \\
\text { values towards professional ethics -, our objective was to discover whether the personnel members } \\
\text { themselves consider that the professional ethical dimension in general, and the professional teaching } \\
\text { ethics in particular, are essential to be a good teaching professional. In this article, we present the } \\
\text { elaboration and validation process of the questionnaire that has been used, with which we hope to } \\
\text { make some contribution towards clarifying aspects related with both professional teaching ethics and } \\
\text { the ethics of professions within the university environment } \\
\text { A partir de la investigación que hemos realizado sobre "Análisis comparado de las actitudes y valores } \\
\text { del profesorado universitario ante la ética profesional", con el objetivo de averiguar si el propio profe- } \\
\text { sorado considera que la dimensión ética profesional en general, y la ética profesional docente, en par- } \\
\text { ticular, son esenciales para ser un buen profesional de la docencia, presentamos, en este artículo, el } \\
\text { proceso de elaboración y validación del cuestionario que se ha utilizado, con el que esperamos hacer } \\
\text { alguna aportación que nos permita aclarar aspectos relacionados con la ética profesional docente y } \\
\text { con la ética de las profesiones en el ámbito universitario }\end{array}$ \\
\hline $\begin{array}{l}\text { Keywords } \\
\text { Descriptores }\end{array}$ & $\begin{array}{l}\text { Instruments validation, attitudes, values, professional ethics, professional teaching ethics, University, } \\
\text { Exploratory Factorial Analysis } \\
\text { Validación de instrumentos, actitudes, valores, ética profesional, ética profesional docente, Universi- } \\
\text { dad, Análisis Factorial exploratorio }\end{array}$ \\
\hline $\begin{array}{l}\text { Institution / } \\
\text { Institución }\end{array}$ & Universidad de Valencia, Universidad Jaime I de Castellón (Spain) \\
\hline $\begin{array}{l}\text { Publication } \\
\text { site /Dirección }\end{array}$ & http://www.uv.es/RELIEVE \\
\hline $\begin{array}{l}\text { Language / } \\
\text { Idioma }\end{array}$ & Spanish (Title, abstract and keywords in English ) \\
\hline
\end{tabular}

\section{Revista ELectrónica de Investigación y $\mathbf{E V}$ aluación Educativa (RELIEVE)}

[ ISSN: 1134-4032 ]

(C) Copyright, RELIEVE. Reproduction and distribution of this articles it is authorized if the content is no modified and their origin is indicated (RELIEVE Journal, volume, number and electronic address of the document).

(C) Copyright, RELIEVE. Se autoriza la reproducción y distribución de este artículo siempre que no se modifique el contenido y se indique su origen (RELIEVE, volumen, número y dirección electrónica del documento). 